\title{
On the requirements to control neoclassical tearing modes in burning plasmas
}

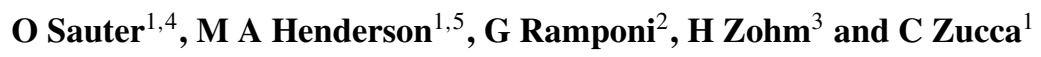 \\ ${ }^{1}$ Ecole Polytechnique Fédérale de Lausanne (EPFC), Centre de Recherches en Physique des \\ Plasmas, Association EURATOM-Confédération Suisse, Station 13, CH-1015 Lausanne, \\ Switzerland \\ 2 Istituto di Fisica del Plasma, EURATOM-ENEA-CNR Association, 20125 Milano, Italy \\ 3 IPP-Garching, Max Planck-Institute für Plasmaphysik, D-85748 Garching, Germany \\ E-mail: olivier.sauter@epfl.ch
}

Received 11 September 2009, in final form 16 November 2009

Published 18 January 2010

Online at stacks.iop.org/PPCF/52/025002

\begin{abstract}
Neoclassical tearing modes (NTMs) are magnetic islands which increase locally the radial transport and therefore degrade the plasma performance. They are self-sustained by the bootstrap current perturbed by the enhanced radial transport. The confinement degradation is proportional to the island width and to the position of the resonant surface. The $q=2$ NTMs are much more detrimental to the confinement than the $3 / 2$ modes due to their larger radii. NTMs are metastable in typical scenarios with $\beta_{\mathrm{N}} \geqslant 1$ and in the region where the safety factor is increasing with radius. This is due to the fact that the local perturbed pressure gradient is sufficient to self-sustain an existing magnetic island. The main questions for burning plasmas are whether there is a trigger mechanism which will destabilize NTMs, and what is the best strategy to control/avoid the modes. The latter has to take into account the main aim which is to maximize the $Q$ factor, but also the controllability of the scenario. Standardized and simplified equations are proposed to enable easier prediction of NTM control in burning plasmas from present experimental results. The present expected requirements for NTM control with localized electron cyclotron current drive (ECCD) in ITER are discussed in detail. Other aspects of the above questions are also discussed, in particular the role of partial stabilization of NTMs, the possibility to control NTMs at small size with little ECH power and the differences between controlling NTMs at the resonant surface or controlling the main trigger source, for the standard scenario namely the sawteeth. It is shown that there is no unique best strategy, but several tools are needed to most efficiently reduce the impact of NTMs on burning plasmas.
\end{abstract}

(Some figures in this article are in colour only in the electronic version)

\footnotetext{
4 Author to whom any correspondence should be addressed.

5 Present address: ITER Organization, St Paul lez Durance, France.
} 


\section{Introduction}

Neoclassical tearing modes (NTMs) have been observed in many tokamaks $[1,2]$ and in particular in H-modes (high confinement modes) with monotonic safety factor $(q)$ profiles. This is due to the low marginal beta above which NTMs are metastable [3]. In H-modes, the bootstrap fraction is increased and the perturbed bootstrap fraction due to the localized island is typically sufficient to sustain the island. The bootstrap current density is perturbed because of the local flattening of the pressure profile within the island, which also leads to a degradation of the confinement. The latter can be well estimated by the belt model [4], yielding a relation between the island full width $w$ and the degradation of the energy confinement time $\tau_{e}$ :

$$
\frac{\Delta \tau_{e}}{\tau_{e}}=\Delta_{\tau} \frac{w_{\mathrm{sat}}}{a}, \quad \text { with } \quad \Delta_{\tau}=4 \frac{\rho_{\mathrm{s}}^{3}}{a^{3}},
$$

where $w_{\text {sat }}$ is the saturated island width, $\rho_{\mathrm{s}}$ the radius of the resonant surface and $a$ the plasma minor radius. NTMs can easily have island widths of $10 \%$ of the minor radius. With such a width and if the mode is localized near mid-radius, the confinement degradation is of the order of $5 \%$. However, if the mode is near $\rho_{\mathrm{s}} / a=0.8$, the degradation is about $20 \%$. This is why $2 / 1$ NTMs, at $q=2$, have much stronger impact on the plasma performance and clearly need to be avoided. Higher $m / n$ modes, where $m, n$ are the poloidal and toroidal mode numbers, respectively, are located closer to the center and therefore have smaller impact on the global plasma performance. This is why one might allow such modes to exist, although even $4 / 3$ modes have been observed to lead up to $10 \%$ degradation in the JET tokamak [5]. Note that the island size can increase significantly if the mode locks and therefore lead to larger confinement degradation [6-8].

Since NTMs are driven by a deficit of current density within the island, the easiest way to stabilize them is by adding localized current with electron cyclotron current drive (ECCD) in the island [9-18]. Recently, a cross-machine comparison of ECCD stabilized NTMs has allowed the prediction of the typical behavior expected in ITER [19]. It is shown that a driven current density of the order of the local bootstrap current density should be sufficient to fully stabilize the mode, $j_{\mathrm{cd}} / j_{\mathrm{bs}} \sim 1$, depending on the assumed model for the stabilizing mechanism at a small island size as will be discussed below. Such a criterion is important for the design of the EC launcher in ITER [20-22]. The range of validity of such a criterion is also important and it will be discussed in detail in section 4 . The aiming accuracy and related issues, as well as the question of the 2/1 mode locking, have been analyzed in [7] and will not be analyzed in details here, except with regard to the global strategy for NTM mitigation.

Another important aspect is the question of the existence of NTMs in burning plasmas and the onset criteria. It has been demonstrated in JET that the main trigger mechanism in low beta plasmas is the sawtooth crash [23]. It is also shown that crashes after a long sawtooth period easily trigger NTMs, even at a very low beta near the marginal beta limit. Since fast particles are very efficient to stabilize sawteeth [24,25], it is assumed here that the main condition for the existence of NTMs in sawtoothing burning plasmas will be related to the sawtooth activity. Therefore, the control of sawteeth is inherently part of any NTM control strategy. This will be discussed in section 6 . Note that for the other scenarios, hybrid and advanced, NTMs typically occur at much higher $\beta_{\mathrm{N}}$ values, close to the ideal limit, and both $\beta_{\mathrm{N}}$ and $q$ profiles influence the onset conditions.

Other aspects related to NTMs and plasma performance are important. First the optimisation should be related to the factor $Q$, the ratio between the fusion and the auxiliary power. This factor characterizes the overall performance of a burning plasma. Since the stabilization of NTMs with ECCD tends to decrease $Q$, by increasing the auxiliary power, an optimum $Q$ might be obtained with partial stabilization of the NTM. Another parameter is the 
relation between the time required to fully stabilize an NTM and the sawtooth period (or rather the time between successive NTM triggers). Finally, the possibility to use pre-emptive ECCD (ECCD applied before the NTM onset) to optimize the power required for NTM stabilization will also be discussed.

The paper is organized as follows, in section 2 we present the main operational diagram relevant for NTM control in burning plasmas, namely the dependence of $Q$ on additional EC power. In section 3 the main equations for comparison with experiments and for the basis for predictions are presented, in section 4 the criteria $\eta_{\mathrm{NTM}}=j_{\mathrm{cd}} / j_{\mathrm{bs}}$ for full stabilization are analyzed, in section 5 predictions for $Q$ and partial stabilization are presented and in section 6 the strategy with respect to sawteeth activity and pre-emptive ECCD is discussed. Section 7 concludes this paper.

\section{Burning plasma conditions}

In order to discuss the best strategies for NTM control, we use the effects of NTMs and/or of the auxiliary power required to stabilize them on the factor $Q$ as a global measure of scenario performance. We use a simplified model to determine the burning temperature and total pressure for a given auxiliary power and island width. We start from the so-called scenario 2 of ITER [1], which is the baseline scenario for reaching $Q=10$ in a sawtoothing ELMy $\mathrm{H}$-mode. The main parameters of interest are $R_{0}=6.2 \mathrm{~m}, a=2 \mathrm{~m}, I_{\mathrm{p}}=15 \mathrm{MA}, B_{0}=5.3 \mathrm{~T}$, $V=830 \mathrm{~m}^{3}, \tau_{E 0}=3.7 \mathrm{~s}, Z_{\text {eff }}=1.7, P_{\mathrm{NBI}}=40 \mathrm{MW}, P_{\alpha}=80 \mathrm{MW}$ and $P_{\mathrm{Brem}}=21 \mathrm{MW}$. The scaling law assumed in this case yields $\tau_{E} \sim P_{\mathrm{L}}^{-e_{\mathrm{P}}}$ with $e_{\mathrm{P}}=0.69$ and $P_{\mathrm{L}}$ the loss power [1]. The fusion power is given by $P_{\mathrm{f}}=5 P_{\alpha}$ with

$$
\begin{aligned}
& P_{\alpha}=\gamma_{\alpha} 1.5 \times 10^{-6} p_{\mathrm{keV}}^{2} R\left(T_{\mathrm{keV}}\right) V(\mathrm{MW}), \\
& R\left(T_{\mathrm{keV}}\right)=29.84 T_{\mathrm{keV}}^{2.5} \exp \left[-\frac{\left(T_{\mathrm{keV}}+0.11\right)^{0.45}}{0.43}\right],
\end{aligned}
$$

using a useful fit for the reactivity $R$ and where $p_{\mathrm{keV}}=1.6 n_{19} T_{\mathrm{keV}}$ is the total pressure with $n_{19}$ expressed in $\left[10^{19} \mathrm{~m}^{-3}\right]$ and $T$ in $[\mathrm{keV}]$. The total thermal energy is given by

$$
W_{E}=\gamma_{E} 3.84 \times 10^{-3} f_{\mathrm{pe}} n_{19} T_{\mathrm{keV}} V(\mathrm{MJ})=P_{\mathrm{L}} \tau_{E},
$$

with $f_{\mathrm{pe}}=p / p_{\mathrm{e}}$ and where $\tau_{E}$ can be written as follows using the baseline parameters:

$$
\tau_{E}=\tau_{E 0}\left(\frac{P_{\mathrm{L} 0}}{P_{\mathrm{L}}}\right)^{e_{\mathrm{P}}}\left(1-\Delta_{\tau} w / a\right),
$$

with $w$ the full island width and $\Delta_{\tau}$ given in equation (1). For the effective total heating power, we take into acount Bremsstrahlung radiation and the fact that any off-axis additional power is located in a bad confinement region. Therefore, we weight its contribution by a profile effect. Assuming steady state we obtain

$$
P_{\mathrm{L}}=P_{\alpha}+P_{\mathrm{NBI}}+\left(1-\frac{\rho_{\mathrm{s}}^{2}}{a^{2}}\right) P_{\mathrm{ec}}-P_{\mathrm{Brem}} .
$$

In our case, given the position of the $2 / 1$ surface, since the $2 / 1$ mode is the main mode degrading plasma performance, we assume $1-\rho_{\mathrm{s}}^{2} / a^{2} \approx 0.5$. The radiation term is important to limit the benefits at large temperatures and is taken as follows:

$$
P_{\text {Brem }}=\gamma_{\mathrm{B}} 47.4 \times 10^{-6} Z_{\text {eff }} n_{19}^{2} V \sqrt{T_{\mathrm{keV}}} .
$$


The parameters $\gamma_{\alpha}, \gamma_{E}, \gamma_{\mathrm{B}}$ are introduced to take into account the profile effects (since the plasma parameters in the above equations are taken at the plasma center). Assuming a flat density profile and $T(\rho) \sim\left(1-\rho^{2}\right)$ one gets

$$
\gamma_{\alpha}=0.19, \gamma_{E}=0.5, \gamma_{\mathrm{B}}=0.67
$$

From equation (4) one obtains:

$$
P_{\mathrm{L}}=\left[\frac{\gamma_{E} 2.410^{-3} f_{\mathrm{pe}} n_{19} T_{\mathrm{keV}} V}{\tau_{E 0} P_{\mathrm{L} 0}^{e_{\mathrm{p}}}\left(1-\Delta_{\tau} w / a\right)}\right]^{\frac{1}{1-e_{\mathrm{p}}}},
$$

which highlights the sensitivity on the power exponent in the scaling law. The burning temperature is then obtained from equations (3), (6), (7) and (9). The measure of the global burning plasma performance, $Q=P_{\mathrm{f}} /\left(P_{\mathrm{NBI}}+P_{\mathrm{ec}}\right)$, is then obtained from equation (3) at this temperature. We have adjusted the factor $f_{\mathrm{pe}}=p / p_{\mathrm{e}}$, total pressure to electron pressure, to 1.83 so as to essentially recover the standard steady-state conditions with no modes $(w=0)$ :

$$
\begin{aligned}
P_{\alpha}=80 \mathrm{MW}, \quad Q & =10, \quad T_{\text {burn }} \approx 20 \mathrm{keV}, \quad \beta_{\mathrm{N}} \approx 1.8, \\
P_{\text {Brem }} & \approx 20 \mathrm{MW},
\end{aligned}
$$

with $P_{\mathrm{NBI}}=40 \mathrm{MW}, P_{\mathrm{L} 0}=99 \mathrm{MW}, \tau_{E 0}=\mathrm{HH} 3.7 \mathrm{~s}$ and $\mathrm{HH}=1$. The HH factor represents any confinement improvement or degradation. With an additional $20 \mathrm{MW}$ of EC power, we obtain $P_{\alpha}=84 \mathrm{MW}$ and $Q=7$. In this way we can determine $P_{\alpha}$ for different $\mathrm{HH}$ values and additional $P_{\mathrm{ec}}$, still assuming no NTMs. The results are shown in figure 1 for $H H$ values equally spaced between 0.75 and 1.25 . This is the operational diagram of interest for this study. We also show the 'anchor' points related to the $3 / 2$ and $2 / 1$ NTMs. If no ECCD is applied, we expect a degradation of $15 \%$ (equation (1)), thus an effective $\mathrm{HH}=0.85$ for the $3 / 2$ mode. Similarly we predict $\mathrm{HH}=0.75$ for the $2 / 1$ mode assuming it does not lock. If it would lock, as predicted in [7] for $w>5-10 \mathrm{~cm}$, the mode would grow to even larger values and the discharge would probably need to be stopped, although recent results show that the locked mode can be controlled as well [26]. This yields $Q=6.9$ with a stationary $3 / 2$ mode and $Q=4.7$ with a $2 / 1$ mode (points $\mathrm{A}$ and $\mathrm{B}$ in figure 1 ). If the modes are fully stabilized with $20 \mathrm{MW}$ and assuming that the EC power needs to be sustained in steady state, we recover $\mathrm{HH}=1$ but at $P_{\mathrm{ec}}=20 \mathrm{MW}$, thus $Q \approx 7$. Of course, if the EC power can be switched off, $Q=10$ is recovered until the next appearance of an NTM if any. Increasing the EC power to stabilize the mode, one will move from points $\mathrm{A}$ or B in figure 1 to point $\mathrm{D}$ (or $\mathrm{C}$ if $10 \mathrm{MW}$ is sufficient to fully stabilize the mode), with a path to be determined and with possible values sketched by the dashed lines. Since the dependence of the saturated island width $w_{\text {sat }}$ on $P_{\text {ec }}$ can be relatively complex, one might find an optimum $Q$ value at lower values of $P_{\mathrm{ec}}$ and thus with a partially stabilized mode. These paths depend on the stabilizing parameters and predicted marginal island widths. They will be calculated in section 5 , but before that, one needs to define the model for calculating $w\left(P_{\mathrm{ec}}\right)$ and to validate it with present experimental results. Depending on effective alignment and assumptions, the mode could be stabilized with $5 \mathrm{MW}$ of ECCD power [7]. In such a case, $\mathrm{CW}$ would be marginally acceptable since it would yield $Q=8.9$.

\section{Standard equations for comparison with experiments}

The main characteristics of NTMs which have been observed experimentally are the proportionality of the saturated island width with poloidal beta, the self-stabilization at a small island width, the confinement degradation (equation (1)), and the efficient stabilization with local ECCD. Other aspects have been observed, but most of them can be included in these 


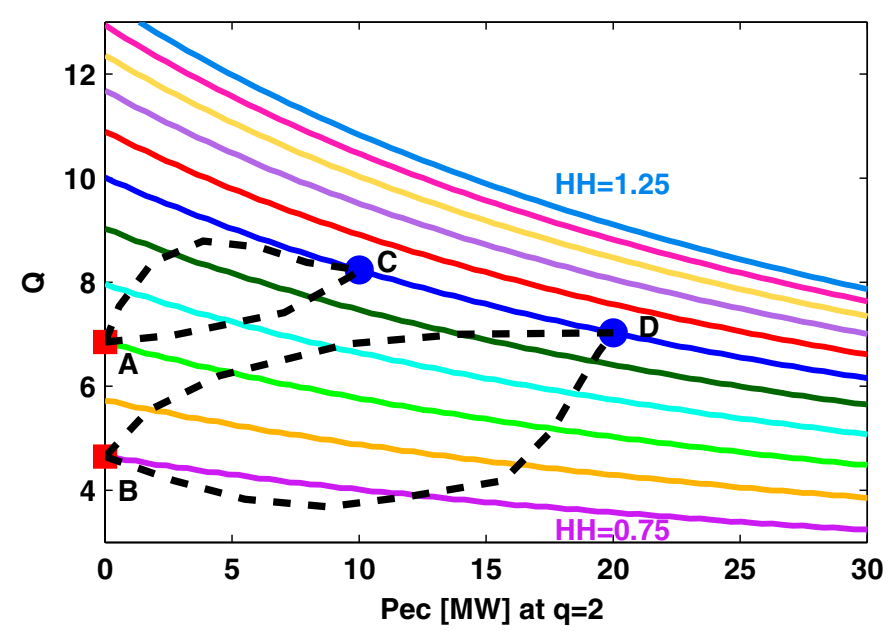

Figure 1. $Q$ versus additional EC power for $\mathrm{HH}$ values between 0.75 and 1.25 . Points A and B mark the predicted steady-state performance with either a $3 / 2$ or a $2 / 1$ NTM, respectively. Points $\mathrm{C}$ and D assume full stabilization with constant $10 \mathrm{MW}$ or $20 \mathrm{MW}$, respectively, and thus are on the curve $\mathrm{HH}=1$. The dashed lines are a sketch of possible stationary operating points with a $3 / 2$ or a $2 / 1$ mode partially stabilized.

global effects. Another important point is the fact that the exact value of the classical tearing parameter related to the total current density profile, $\rho_{\mathrm{s}} \Delta^{\prime}$, is neither easily measured nor well defined. These characteristics are well encapsulated by the modified Rutherford equation. Since $\rho_{\mathrm{s}}\left|\Delta^{\prime}\right|$ is de facto a free parameter, it is better to normalize the equation for the island growth rate by this value and one obtains the following two basic equations [3,27]:

$$
\begin{aligned}
& \frac{\tau_{\mathrm{R}}}{\rho_{\mathrm{s}}^{2}\left|\Delta^{\prime}\right|} \frac{\mathrm{d} w}{\mathrm{~d} t}=-1+\left(1-\frac{\Delta_{\tau} w}{a}\right) \frac{w_{\mathrm{sat} \infty\left(\beta_{p}\right)} w}{w^{2}+w_{\mathrm{marg}}^{2}}-\tilde{\Delta}_{\mathrm{cd}}^{\prime}, \\
& \frac{\tau_{\mathrm{R}}}{\rho_{\mathrm{s}}^{2}\left|\Delta^{\prime}\right|} \frac{\mathrm{d} w}{\mathrm{~d} t}=-1+\left(1-\frac{\Delta_{\tau} w}{a}\right) \frac{w_{\mathrm{sat} \infty\left(\beta_{p}\right)}}{w}\left(1-\frac{w_{\mathrm{marg}}^{2}}{3 w^{2}}\right)-\tilde{\Delta}_{\mathrm{cd}}^{\prime},
\end{aligned}
$$

with

$$
\tilde{\Delta}_{\mathrm{cd}}^{\prime}=c_{j}\left(1-\frac{\Delta_{\tau} w}{a}\right) \frac{w_{\mathrm{sat} \infty}}{w_{\mathrm{cd}}} \frac{j_{\mathrm{cd}}}{j_{\mathrm{bs}}} \eta_{\mathrm{aux}}\left(w / w_{\mathrm{cd}}\right) .
$$

The left-hand side represents the normalized island growth rate, where $\tau_{\mathrm{R}}$ is the resistive time. The first term on the right-hand side is the stabilizing contribution from the equilibrium current density at large island size. The latter is always negative at large island and should depend on $w$ [28]. Here we assume $\rho_{\mathrm{s}} \Delta^{\prime}$ to be constant and negative, which is reasonable if the ratio between the largest saturated width and the marginal island width is not very large. However, a term like $(1-\alpha w)$ should replace the term $(-1)$ if the largest saturated width becomes very large [28].

The second term on the right-hand side is the driving term from the perturbed bootstrap current: $\rho_{\mathrm{s}} \Delta_{\mathrm{bs}}^{\prime}$. It is proportional to $1 / w$ at a large island size [29] and is reduced at a small island size because the effective perturbed bootstrap current is not as large as if the pressure was fully flattened. There are typically two forms which can explain the observed behavior at small island size: equation (11) related to the effect of finite $\chi_{\perp} / \chi_{\|}$[30], and equation (12) related to the effect of the polarization current [31]. The latter has been assumed in the recent 
cross-machine analysis [19]. We propose to keep both options, since they have very different behavior at small $w$, the first one leads to $\rho_{\mathrm{s}} \Delta_{\mathrm{bs}}^{\prime} \sim w$ and the second to $\rho_{\mathrm{s}} \Delta_{\mathrm{bs}}^{\prime} \sim 1 / w-1 / w^{3}$. For full stabilization with ECCD, this is important because it takes place at a small island size. It is argued that using both forms proposed in equations (11) and (12) should span the effective dependence of the growth rate at small island width. The latter can indeed be rather complicated once the effects on the curvature term [32] or of finite orbit width [33], for example, are taken into account. Note that we use the simplest form which still takes the main physical effects. Therefore, for the second model, the frequency dependence is ignored and only the main stabilizing contribution is taken into account with its $w$ dependence. If the latter would decrease due to frequency change, the first model would remain.

The values of $w_{\text {sat } \infty}$ and $w_{\text {marg }}$ determine the strength of the driving term and of the stabilizing contributions at small island, respectively. The first relates to the saturated width at a large island size. Indeed, for $w \gg 1$, both equations give the same saturated island width, without ECCD contribution:

$$
w_{\text {sat }}\left(\mathrm{d} w / \mathrm{d} t=0, j_{\mathrm{cd}}=0, w_{\text {marg }} \cong 0\right)=\frac{w_{\text {sat } \infty}}{1+\Delta_{\tau} \frac{w_{\text {sat } \infty}}{a}},
$$

where the denominator includes self-consistently the reduction in $\beta_{\mathrm{p}}$ due to the NTM and $w_{\text {sat } \infty}$ is the saturated island size without confinement degradation nor the stabilizing terms $\left(w_{\text {sat }} \gg w_{\text {marg }}\right.$, thus the subscript ' $\infty$ '). The latter can be related to the usual terms of the modified Rutherford equation with

$$
w_{\mathrm{sat} \infty}=\rho_{\mathrm{s}} \beta_{\mathrm{p}} \frac{a_{\mathrm{bs}}-a_{\mathrm{ggj}}}{\rho_{\mathrm{s}}\left|\Delta^{\prime}\right|}
$$

where the bootstrap and ggj contributions are defined in [2] and the plasma parameters are taken at the mode onset (or equivalently without the presence of a mode). Once the mode onsets, it is seen experimentally that the global $\beta_{\mathrm{p}}$ decreases and the resultant saturated island

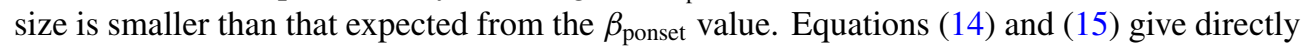
the self-consistent saturated island width and the stationary $\beta_{\mathrm{p}}=\beta_{\text {ponset }} /\left(1+\Delta_{\tau} \frac{w_{\text {sat }}}{a}\right)$. For cross-machine comparison and prediction to ITER, relatively self-similar plasmas need to be considered (here sawtoothing, ELMy H-modes for scenario 2) and the global $\beta_{\mathrm{p}}$ is used. This has proven useful and robust in analyzing and predicting NTMs behavior in AUG [8], DIII-D [34], JET [3,35,36], MAST [37] and TCV [28] with the same method as defined in [2,3]. Using ITER scenario 2 parameters, one obtains $w_{\text {sat } \infty}=32 \mathrm{~cm}$ from equation (15) which leads to a predicted effective stationary saturated island width of $24 \mathrm{~cm}$ (equation (14)) once the self-consistent confinement degradation is taken into account. This finally gives an effective confinement degradation of $25 \%$. For the $3 / 2$ mode, we get $w_{\text {sat } \infty}=25 \mathrm{~cm}$ and $w_{\text {sat }}=21 \mathrm{~cm}$ from equation (14) for an effective confinement degradation of $15 \%$. For the $2 / 1$ mode the expected saturated width without EC is larger than the width above which the mode is predicted to lock [7]. We shall discuss the implications in the last sections. The value here is used to determine the characteristics near full stabilization.

The marginal island width $w_{\text {marg }}$ is the island width at the maximum growth rate, without ECCD contribution. Actually, at $\max (\mathrm{d} w / \mathrm{d} t)$ we have $w \simeq w_{\operatorname{marg}}\left(1-\Delta_{\tau} w_{\text {marg }} / a\right)$, but the confinement degradation at $w=w_{\text {marg }}$ is less than $5 \%$. Thus, the main characteristics of the modified Rutherford equation are fully determined, without ECCD, once only two parameters are determined, namely $w_{\text {sat } \infty}$ and $w_{\text {marg }}$. This is shown in figure $2(a)$, where examples of equations (11) and (12) are shown, with $j_{\text {cd }}=0$. Dashed-dotted lines show the island growth rate without the self-consistent confinement degradation (i.e. assumes $\Delta_{\tau}=0$ ) and therefore the saturated island width is at $w=w_{\text {sato }}$ (stable point with $\mathrm{d} w / \mathrm{d} t=0$ ). On the other hand, including the $\Delta_{\tau}$ effect (solid lines) modifies the curves at large $w$ and leads to the smaller 

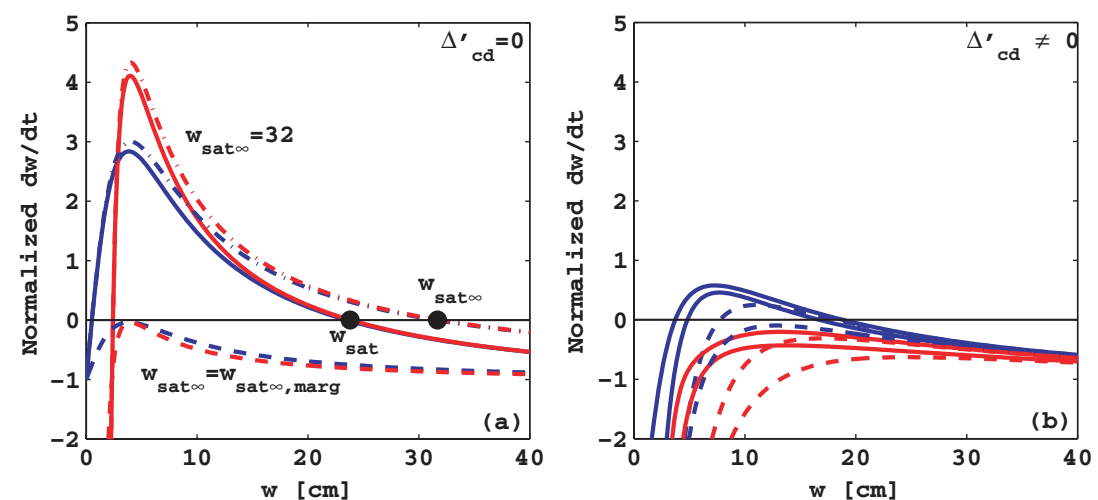

Figure 2. RHS of equation (11) (blue online) or equation (12) (with a sharper drop at small $w$, red online) with $w_{\text {marg }}=4 \mathrm{~cm}, w_{\mathrm{sat} \infty}=32 \mathrm{~cm}$ and $\Delta_{\tau}=2.05$. (a) $\Delta_{\mathrm{cd}}^{\prime}=0$, with (solid) and without (dashed-dotted) the term $\Delta_{\tau}$. The dashed lines are obtained with equation (17). (b) Same as solid lines of $(a)$ including $\Delta_{\text {cd }}^{\prime}$ assuming the $50 \%$ or $\mathrm{CW}$ with $w_{\mathrm{cd}}=2.5 \mathrm{~cm}$ (dashed) or $5 \mathrm{~cm}$ (solid) and 13.3 MW.

effective saturated island width $w_{\text {sat }}$ (equation (14)). This is due to the effect of the island on confinement. If a mode onsets at $\beta_{\mathrm{p}}=0.66$, it will degrade the confinement, $\beta_{\mathrm{p}}$ will decrease and the mode will not grow to the same size as if additional power would maintain $\beta_{\mathrm{p}}=0.66$. This stationary effective $w_{\text {sat }}$ is directly obtained with the term $\Delta_{\tau}$ and fixed onset conditions determined by $w_{\text {sat } \infty}$. The curves correspond to the predicted $2 / 1$ mode in ITER $Q=10$ scenario with $w_{\text {sat } \infty}=32 \mathrm{~cm}, w_{\text {sat }}=24 \mathrm{~cm}, w_{\text {marg }}=4 \mathrm{~cm}$ and thus $\Delta_{\tau} w_{\text {sat }} / a=25 \%$. It is also interesting to note the value of $w_{\text {sat } \infty}$ required such that the maximum growth rate is zero, which determines the marginal beta limit [3]:

$$
\begin{array}{lr}
w_{\text {sat } \infty \text { marg }}=2 w_{\text {marg }} & \text { for equation (11), } \\
w_{\text {sat } \infty, \operatorname{marg}}=1.5 w_{\operatorname{marg}} & \text { for equation (12). }
\end{array}
$$

The dashed curves in figure $2(a)$ have been obtained with $w_{\text {sat } \infty}$ set to the above values. In this way, the ratio of $w_{\text {sat } \infty}$, measured with the onset $\beta_{\mathrm{p}}$, with $2 w_{\text {marg }}$ or $1.5 w_{\text {marg }}$ gives the hysteresis factor and is similar to the ratio $\beta_{\mathrm{p} \text {,onset }} / \beta_{\mathrm{p} \text {,marg }}$.

Experimentally, these two parameters, $w_{\text {sat } \infty}$ and $w_{\text {marg }}$, are easily measured. The first one, $w_{\text {sat } \infty}$, is measured from the island width $w_{\text {sat }}$ when a sufficient $\beta_{\mathrm{p}}$ is maintained. In this case, the effect of the confinement degradation is not negligible and $\Delta_{\tau}$ in equation (14) should be taken into account, as seen from figure $2(a)$. The second parameter, $w_{\text {marg }}$, is obtained from slow power ramp-down experiments $[3,8]$ from the island width when the mode quickly selfstabilizes. Note that a very slow decrease in $\beta_{\mathrm{p}}$ is required to accurately measure $w_{\text {marg }}$ [3]. The ratio of $w_{\text {sat } \infty} / w_{\text {marg }}$ should be of the order of 5 or larger, to ensure an accurate measure of the first parameter. Note that this is often not the case in ECCD experiments, where the saturated island width before the ECCD is applied is often relatively small.

The last term on the right-hand side of equations (11) and (12) represents the contribution due to localized ECCD, $\tilde{\Delta}_{\mathrm{cd}}^{\prime}=\Delta_{\mathrm{cd}}^{\prime} /\left|\Delta^{\prime}\right|$ (equation (13)). Here we assume perfect alignment, while misalignment has been taken into account in $[7,19]$. The coefficient $c_{j}$ is used to fit the experimental results with the above equation. The driven current is assumed to be of the form $j=j_{\mathrm{cd}} \exp \left[-4\left(\rho-\rho_{\mathrm{s}}\right)^{2} / w_{\mathrm{cd}}^{2}\right]$, such that $j_{\mathrm{cd}}$ is the peaked current density and $w_{\mathrm{cd}}$ is the $1 / e$ full width. The function $\eta_{\text {aux }}\left(w / w_{\mathrm{cd}}\right)$ is related to the stabilizing contribution of the parallel 
current driven within the island and has typically the following dependence on $w[13-15,18]$ :

$$
\begin{aligned}
& \eta_{\text {aux }, \mathrm{cw}}\left(x=\frac{w}{w_{\mathrm{cd}}}\right)=\frac{1}{1+2 x^{2} / 3}, \\
& \eta_{\mathrm{aux}, 50 \%}\left(x=\frac{w}{w_{\mathrm{cd}}}\right)=\frac{1.8}{x^{2}} \tanh \left(\frac{x}{2.5}\right) .
\end{aligned}
$$

The fits are taken from [18], with a factor $1 / 2$ for equation (19) to take into account the effective total driven current and normalized such that $\eta_{\text {aux }} \cong 1$ for $w \cong w_{\text {cd }}$. The first function, equation (18), assumes a localized CW deposition and the second, equation (19), a localized deposition near the O-point with $50 \%$ modulation. The effect of adding the last term on the right-hand side of equations (11) and (12) is shown in figure $2(b)$. An important characteristic is that the $w$ dependence of $\eta_{\text {aux }}$ is not necessarily the same as the other contributions. Therefore, the maximum growth rate might shift to larger values than $w_{\text {marg }}$, which might have important effects when comparing various current density profile widths as will be discussed below. This is clearly seen in figure $2(b)$ where we have used the solid line case of figure 2(a) and added $\Delta_{\text {cd }}^{\prime}$ with $j_{\mathrm{cd}} / j_{\mathrm{bs}}$ corresponding to the prediction for ITER with $13.3 \mathrm{MW}$ and $w_{\mathrm{cd}}=2.5$ or $5 \mathrm{~cm}$. We see that we can essentially stabilize the mode and that the island width will self-stabilize at about $w=10 \mathrm{~cm}($ when $\max (\mathrm{d} w / \mathrm{d} t)=0)$. This is indeed 2.5 times larger than $w_{\text {marg }}$ and is a realistic situation.

Another form for $\eta_{\mathrm{aux}, \mathrm{cw}}$ has been used in [27], $\eta_{\mathrm{aux}, \mathrm{fs}}$ corresponding to equation (20) of [18], but this would not change much the results presented here. It should be noted that in [18], when assuming a flux surface current density, the author neglected the fact that the phase $\alpha_{0}$ in equation (19) still depends on the flux surface label $\psi$. This will in turn define the effective current density inside the island and gives values for $\eta_{\text {aux }}$ in between $\eta_{\text {aux,cw }}$ and $\eta_{\text {aux,fs }}$, depending on the assumed function $\alpha_{0}(\psi)$. It is therefore better, for conservative assumptions, to take the form of equation (18) which was first calculated in [15]. The differences lie within the error bar of present experimental results and the related predictions that one can draw.

\section{Criteria for $\eta_{\mathrm{NTM}} \equiv j_{\mathrm{cd}} / j_{\mathrm{bs}}$}

Due to the high probability for ITER to have NTMs in its standard scenario [23] and the efficient stabilization obtained with ECCD [9-12], ITER will be equipped with EC upper launchers dedicated mainly to NTM stabilization. The design of such a launcher tries to maximize the peaked $j_{\mathrm{cd}}$ while minimizing the value of $w_{\mathrm{cd}}$. However, to evaluate whether a given design is predicted to be able to fully stabilize NTMs in ITER and moreover at what power level, it is useful to have a criterion for $\eta_{\mathrm{NTM}} \equiv j_{\mathrm{cd}} / j_{\mathrm{bs}}$. At present, a criterion of $\eta_{\mathrm{NTM}} \geqslant 1.2$ is used [21], based on $[19,20]$. The latter is obtained assuming $50 \%$ modulation and the polarization model, equation (12). It does not take into account the deposition width, $w_{\text {cd }}$, nor the marginal island width $w_{\text {marg }}$. Here, we analyze the effects of these two parameters, in addition to assuming different models for the stabilizing terms at small island width and $\mathrm{CW}$ versus $50 \%$ modulation. The aim is to obtain a more complete definition of the criteria for $\eta_{\mathrm{NTM}}$ and a well-defined scheme to analyze the performance of a given launcher design.

\subsection{Basic ITER parameters used for the study}

Before analyzing the criteria for $\eta_{\mathrm{NTM}}$, one has to determine the basic plasma parameters required in equations (11) and (12). That is, the expected values of $w_{\text {sat } \infty}$ and $w_{\text {marg }}$ and the 
Table 1. Parameters in equations (11) and (12) used to determine the values of $c_{j}$ required in both equations, respectively, with $w_{\text {sat } \infty, \chi}$ from equation (11) and $w_{\text {sat } \infty \text {,pol }}$ from equation (12).

\begin{tabular}{lllclr}
\hline Machine & $w_{\text {marg }}$ & $w_{\text {sat } \infty, \chi}$ & $w_{\text {sat } \infty, \text { pol }}$ & $j_{\mathrm{cd}} / j_{\mathrm{bs}}$ & $w_{\mathrm{cd}}$ \\
\hline AUG & $1.5-1.8$ & 3.7 & 2.9 & 3.1 & 1.1 \\
DIII-D & 2.5 & 6.6 & 5.8 & 0.9 & 2.5 \\
JET & 4 & 9.1 & 8.3 & 1.2 & 3.8 \\
JT-60U & $4-5$ & 12 & 10.5 & 1.2 & 11.2 \\
\hline
\end{tabular}

value of $c_{j}$ to fit the present experiments. The value of $\Delta_{\tau}$ is given from the equilibrium used for the ray-tracing simulations. First the coefficient $c_{j}$ has been determined using the same method as in [20]. We have obtained the relevant parameters to reproduce the experimental results presented in [19], using $w_{\mathrm{cd}}=6 \delta_{\mathrm{ec}} / 5$. They are given in table 1 . Then, a coefficient $c_{j}$ is obtained such that all experiments in which full stabilization is realized lead to $\max (\mathrm{d} w / \mathrm{d} t)<0$. Since there are large uncertainties in these early cross-machine comparisons, we have taken 'best estimates' for the relevant coefficients. We have taken a conservative value of $c_{j}=0.5$ to be used with equation (11) and a value of $c_{j}=1$ for equation (12). Note that we have not considered the effects of local heating within the island, as has been recently observed in TEXTOR [38], nor the effect of $j_{\mathrm{cd}}$ on $\Delta^{\prime}$ [39]. These effects should not be dominant in ITER, with good alignment, and they are in fact taken into account, to first order, with the coefficient $c_{j}$. In addition to this coefficient, one should take into account the misalignment in the various experiments. However, the latter is not yet well determined, therefore the method to determine $c_{j}$ proposed here can be seen as purposedly conservative for predictions to burning plasmas. We also assume that these coefficients, obtained from $3 / 2$ mode stabilization experiments, are also valid for $2 / 1$ modes.

We now need to determine the plasma parameters expected in ITER. As mentioned earlier, we only need to predict two parameters, namely $w_{\text {sat } \infty}$ and $w_{\text {marg }}$. The latter has been predicted to be near $2 \mathrm{~cm}$ in [19] and 2-6 cm in [3]. We shall use three values, 2, 4 and $6 \mathrm{~cm}$ in order to study its influence on the predicted results. On the other hand, the value of $w_{\text {sat } \infty}$ has not yet been studied systematically across machines, although it would also allow a good test of the theory by comparing with equation (15). Therefore, we have used the same method as described in [2] and which has been used to compare with DIII-D [34], JET [3, 35, 36], MAST [37] and TCV [28]. In each case, the saturated island size was relatively well predicted using global plasma parameters and the formulae in [2]. Using the parameters predicted for the standard scenario in ITER, scenario 2 [1] with $\beta_{\mathrm{p}}=0.66$ and $\beta_{\mathrm{N}}=1.8$, we obtain for $q=2$, a value of $w_{\text {sat } \infty}=32 \mathrm{~cm}$ and $\rho_{\mathrm{s}} / a=0.8$ with $a=200 \mathrm{~cm}$. The latter yields $\Delta_{\tau}=2.05$ and an effective saturated island from equation (14) of $w_{\text {sat }, 2 / 1, \text { ITER }} \simeq 24 \mathrm{~cm}$. Thus, one would have an island of $12 \%$ the minor radius and a confinement degradation of $25 \%$ with $\beta_{\mathrm{N}}=1.8$, which looks reasonable when compared with the present experimental results with rotating 2/1 modes.

The latest design for the ITER upper launcher $[22,40]$ has a peaked current density with $13.3 \mathrm{MW}$ of $j_{\mathrm{cd}} \simeq 0.2 \mathrm{MA} \mathrm{m}^{-2}$, such that $\eta_{\mathrm{NTM}} \simeq 2.7$, and a width $w_{\mathrm{cd}} \simeq d / \sqrt{\kappa} \simeq 2.35 \mathrm{~cm}$, where $d$ is the width defined with respect to the area [40]. The launcher is made of two rows and the other row has a slightly larger value of $w_{\mathrm{cd}}$. This is why we have analyzed the results assuming $w_{\mathrm{cd}}=2.5,5$ and $10 \mathrm{~cm}$ in order to see the effects of larger deposition widths. Usually the total driven current is relatively constant, under similar launching conditions. Therefore, when considering various widths, one should keep $\left(\eta_{\mathrm{NTM}} w_{\mathrm{cd}}\right)$ constant. We shall use $\eta_{\mathrm{NTM}} w_{\mathrm{cd}}=6.3$ to analyze the present launcher design, which corresponds to the value predicted with $13.3 \mathrm{MW}(2 / 3$ of the available power). 
Table 2. Values of $\eta_{\text {NTM }}$ expected to stabilize 2/1 NTMs on ITER. The first three rows correspond to different values of $w_{\text {marg. }}$. The columns span the values of $w_{\mathrm{cd}}=2.5,5$ and $10 \mathrm{~cm}$, as well as the two models considered using equation (11) for the columns labeled ' $\chi_{\perp}$ ' and using equation (12) for the columns labeled 'pol'. The last two rows yield the average value per model and then per current density width $w_{\text {cd }}$. Table for the CW case, using equation (18).

\begin{tabular}{|c|c|c|c|c|c|c|}
\hline \multirow[b]{2}{*}{$w_{\text {marg }}$} & \multicolumn{2}{|c|}{$w_{\mathrm{cd}}=2.5 \mathrm{~cm}$} & \multicolumn{2}{|c|}{$w_{\mathrm{cd}}=5 \mathrm{~cm}$} & \multicolumn{2}{|c|}{$w_{\mathrm{cd}}=10 \mathrm{~cm}$} \\
\hline & $\chi_{\perp}$ & $\chi_{\text {pol }}$ & $\chi_{\perp}$ & $\chi_{\text {pol }}$ & $\chi_{\perp}$ & $\chi_{\text {pol }}$ \\
\hline $2 \mathrm{~cm}$ & $3.7 ; 9.25$ & $1.9 ; 4.75$ & $2.5 ; 12.5$ & $1.7 ; 8.5$ & $4.4 ; 44$ & $3.1 ; 31$ \\
\hline $4 \mathrm{~cm}$ & $3.2 ; 8$ & $1.8 ; 4.5$ & $1.9 ; 9.5$ & $1.1 ; 5.5$ & $2.0 ; 20$ & $1.5 ; 15$ \\
\hline $6 \mathrm{~cm}$ & $2.6 ; 6.5$ & $1.7 ; 4.25$ & $1.5 ; 7.5$ & $1.0 ; 5$ & $1.3 ; 13$ & $1.0 ; 10$ \\
\hline$\eta_{\mathrm{NTM}} ; \eta_{\mathrm{NTM}} w_{\mathrm{cd}}$ & $3.17 ; 7.93$ & $1.8 ; 4.5$ & $1.97 ; 9.85$ & $1.27 ; 6.35$ & $2.57 ; 25.7$ & $1.87 ; 18.7$ \\
\hline$\eta_{\mathrm{NTM}} ; \eta_{\mathrm{NTM}} w_{\mathrm{cd}}$ & \multicolumn{2}{|c|}{$2.49 ; 6.2$} & \multicolumn{2}{|c|}{$1.62 ; 8.1$} & \multicolumn{2}{|c|}{$2.22 ; 22.2$} \\
\hline$\eta_{\mathrm{NTM}} w_{\mathrm{cd}}$ & \multicolumn{4}{|c|}{7.15} & & \\
\hline
\end{tabular}

Table 3. Same as table 2 but assuming 50\% modulation in the O-point, equation (19).

\begin{tabular}{|c|c|c|c|c|c|c|}
\hline \multirow[b]{2}{*}{$w_{\text {marg }}$} & \multicolumn{2}{|c|}{$w_{\mathrm{cd}}=2.5 \mathrm{~cm}$} & \multicolumn{2}{|c|}{$w_{\mathrm{cd}}=5 \mathrm{~cm}$} & \multicolumn{2}{|c|}{$w_{\mathrm{cd}}=10 \mathrm{~cm}$} \\
\hline & $\chi_{\perp}$ & $\chi_{\text {pol }}$ & $\chi_{\perp}$ & $\chi_{\text {pol }}$ & $\chi_{\perp}$ & $\chi_{\text {pol }}$ \\
\hline $2 \mathrm{~cm}$ & $3.0 ; 7.5$ & $1.5 ; 3.75$ & $2.1 ; 10.5$ & $1.1 ; 5.5$ & $2.0 ; 20$ & $1.1 ; 11$ \\
\hline $4 \mathrm{~cm}$ & $2.6 ; 6.5$ & $1.5 ; 3.75$ & $1.7 ; 8.5$ & $1.0 ; 5.0$ & $1.5 ; 15$ & $0.9 ; 9$ \\
\hline $6 \mathrm{~cm}$ & $2.1 ; 5.25$ & $1.4 ; 3.5$ & $1.3 ; 6.5$ & $0.9 ; 4.5$ & $1.1 ; 11$ & $0.8 ; 8$ \\
\hline$\eta_{\mathrm{NTM}} ; \eta_{\mathrm{NTM}} w_{\mathrm{cd}}$ & $2.57 ; 6.43$ & $1.47 ; 3.68$ & $1.7 ; 8.5$ & $1.0 ; 5.0$ & $1.53 ; 15.3$ & $0.93 ; 9.3$ \\
\hline$\eta_{\mathrm{NTM}} ; \eta_{\mathrm{NTM}} w_{\mathrm{cd}}$ & \multicolumn{2}{|c|}{$2.02 ; 5.05$} & \multicolumn{2}{|c|}{$1.35 ; 6.75$} & \multicolumn{2}{|c|}{$1.23 ; 12.3$} \\
\hline$\eta_{\mathrm{NTM}} w_{\mathrm{cd}}$ & & 5.9 & & & & \\
\hline
\end{tabular}

\subsection{The criteria $\eta_{\mathrm{NTM}}$}

Using the above parameters into equation (11) or (12), with different values of $w_{\operatorname{marg}}(2,4$ or $6 \mathrm{~cm})$ and $w_{\mathrm{cd}}(2.5,5$ or $10 \mathrm{~cm})$, we can determine the value of $\eta_{\mathrm{NTM}}=j_{\mathrm{cd}} / j_{\mathrm{bs}}$ such that the NTM is unconditionally stable $(\max (\mathrm{d} w / \mathrm{d} t)<0)$. These values are given in tables 2 and 3 , with average values given in the three bottom rows. The value of the product $\eta_{\mathrm{NTM}} w_{\mathrm{cd}}$ is also provided, since it is very useful when comparing various values of $w_{\mathrm{cd}}$. For the present ITER EC upper launcher design [22] with $13.3 \mathrm{MW}$, the expected value of $\eta_{\mathrm{NTM}}$ is $2.52,1.26$ or 0.63 if $w_{\mathrm{cd}}=2.5,5$ or $10 \mathrm{~cm}$, respectively. Looking at tables 2 and 3 , we see that for $w_{\mathrm{cd}}=2.5 \mathrm{~cm}$, in five cases out of six for $50 \%$ modulation and four out of six for $\mathrm{CW}$, the NTM can be stabilized with the present design of the EC launcher. For $w_{\mathrm{cd}}=5 \mathrm{~cm}, 2 / 1$ NTMs can be stabilized in four out of six test cases for both the $\mathrm{CW}$ option and the $50 \%$ modulation. Finally for $w_{\mathrm{cd}}=10 \mathrm{~cm}$, NTMs cannot be stabilized in CW and marginally for 1 case using $50 \%$ modulation (for $w_{\text {marg }}=6 \mathrm{~cm}$ ). Assuming $20 \mathrm{MW}$, thus $\eta_{\mathrm{NTM}} w_{\mathrm{cd}}=9.45$, essentially all cases with $w_{\mathrm{cd}}=2.5 \mathrm{~cm}$ or $5 \mathrm{~cm}$ can be fully stabilized. From tables 2 and 3 , we also see that depending on the model the $2 / 1$ mode would be fully stabilized with EC power between $7 \mathrm{MW}$ and $20 \mathrm{MW}$. Comparing $w_{\mathrm{cd}}=5 \mathrm{~cm}$ with $10 \mathrm{~cm}$, we clearly see that localization is beneficial since the required $\left(\eta_{\mathrm{NTM}} w_{\mathrm{cd}}\right)$ values decrease by a factor of two to three and the required $\eta_{\mathrm{NTM}}$ is similar or smaller. On the other hand, when using $2.5 \mathrm{~cm}$ instead of $5 \mathrm{~cm}$, the required values of $\eta_{\mathrm{NTM}}$ increase while the required $\eta_{\mathrm{NTM}} w_{\mathrm{cd}}$ are similar. This is due to the fact that $w_{\mathrm{cd}}$ starts to be of the order of $w_{\text {marg }}$, so there is little gain by reducing it further. Actually, a high degree of radial accuracy is also required for the ECCD deposition location, of the order of $w_{\mathrm{cd}}$ or less [7]. Therefore, a value of $w_{\mathrm{cd}} \approx 5 \mathrm{~cm}$ could actually be an optimum. 


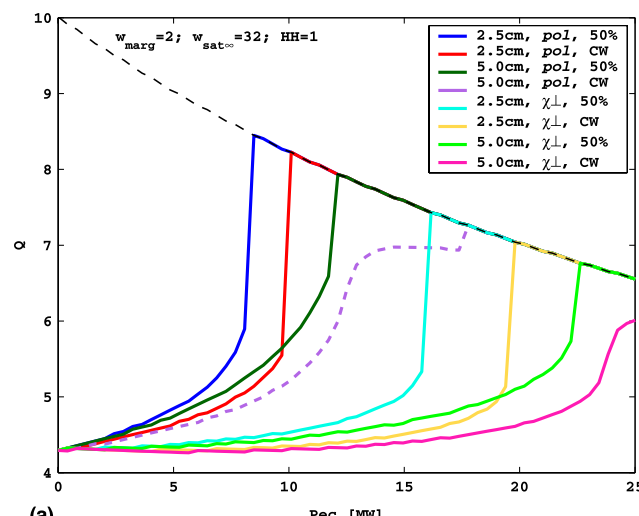

(a)

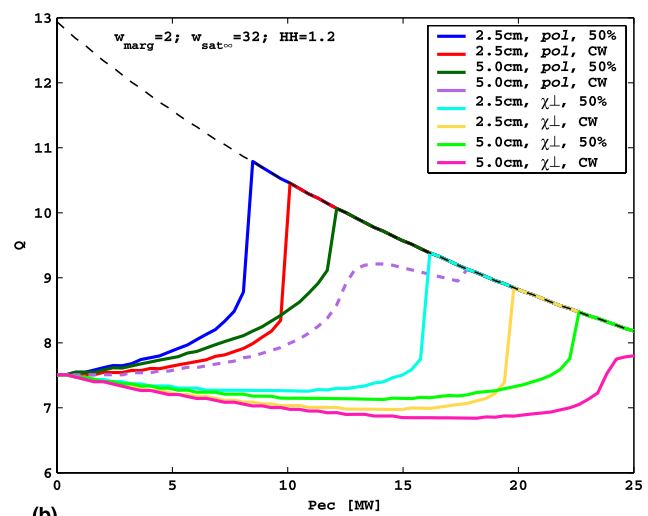

(b)

Figure 3. $Q$ factor obtained with a $2 / 1$ mode controlled with $P_{\mathrm{EC}}$ for various models, assuming $w_{\text {marg }}=2 \mathrm{~cm}, w_{\text {sat } \infty}=32 \mathrm{~cm}$. The required values for full stabilization are given in tables 2 and 3. (a) Assuming $\mathrm{HH}=1,(b)$ with $\mathrm{HH}=1.2$.

Tables 2 and 3 can be used to compare between the stabilizing models. The required values for the ' $\chi_{\text {pol }}$ ' model (equation (12)) are typically $50-70 \%$ of the ones for the ' $\chi_{\perp}$ ' model (equation (11)). However, a large part is due to the lower value of $c_{j}$ used in equation (11) which was very conservative. Using the same value as in equation (12) would lead to similar predicted values in both cases. However, one needs further detailed experiments to better quantify this value. Finally, we can compare the required values assuming $\mathrm{CW}$ (table 2) and $50 \%$ modulation (table 3 ). We see that there are actually no significant differences except for $w_{\text {marg }}=2$, since in this case $w / w_{\text {cd }}$ can be smaller than one, which is the domain where $50 \%$ modulation can be beneficial [12].

The criteria $\eta_{\mathrm{NTM}} \geqslant 1.2$ was essentially based on the 'pol' model, equation (12), and assuming $50 \%$ modulation. With this model, we obtain a similar value, $\eta_{\mathrm{NTM}} \geqslant 1$ from table 3 for the cases with $w_{\text {cd }}=5 \mathrm{~cm}$ and independently of the value of $w_{\text {marg }}$. However if $w_{\mathrm{cd}}=2.5 \mathrm{~cm}$, then $\eta_{\mathrm{NTM}} \geqslant 1.5$ should be used. Therefore, from tables 2 and 3 we see that a more general criteria should be

$$
w_{\mathrm{cd}} \leqslant 5 \mathrm{~cm} \quad \text { and } \quad \eta_{\mathrm{NTM}} w_{\mathrm{cd}} \geqslant 5 \mathrm{~cm} .
$$

\section{5. $Q$ predictions in the presence of finite widths NTMs and EC stabilization}

The parameters given in tables 2 and 3 define the requirements for full stabilization. As discussed in section 2 and shown in figure 1, one should consider the effective paths between no stabilization and full stabilization in order to determine the best strategy. This is performed in this section where we find the effective $Q$ value for a given EC power and current driven, and a resulting saturated island size. In other words we solve equation (11) or (12) to determine the saturated island size which determines through equations (1) and (3)-(9) the resulting $Q$ factor. In this section we assume that the EC power is turned on all along. The case of stabilizing the mode and then switching off the EC power is considered in the next section.

We show in figure 3 the effective paths of a $2 / 1$ NTM in ITER in the operational diagram $Q$ versus EC power. We show the eight cases with $w_{\text {marg }}=2 \mathrm{~cm}$ and $w_{\text {cd }}=2.5$ or $5 \mathrm{~cm}$ corresponding to the top row of tables 2 and 3 . The order in the legend corresponds to 

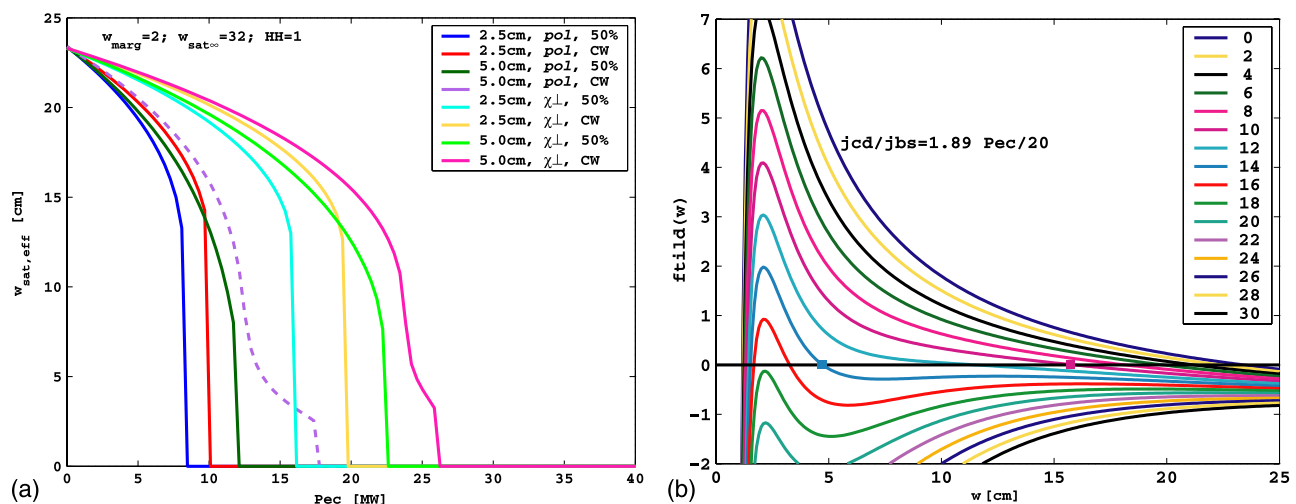

Figure 4. (a) Effective saturated island width with a given $P_{\mathrm{EC}}$ as obtained from equations (11) and (12) corresponding to figure 3. (b) RHS of equation (12) for the case shown with a dashed line in $(a)$ and figure 3 .

increasing required $\left(\eta_{\mathrm{NTM}} w_{\mathrm{cd}}\right)$ values obtained in tables 2 and 3 for full stabilization. For example, for $w_{\mathrm{cd}}=2.5 \mathrm{~cm}, \mathrm{CW}$ and assuming the $\chi_{\perp}$ model, table 2 gives $\eta_{\mathrm{NTM}} w_{\mathrm{cd}}=9.25$. Since the UL provide $\eta_{\mathrm{NTM}} w_{\mathrm{cd}}=6.3$ for $13.3 \mathrm{MW}$, this means that $19.5 \mathrm{MW}$ are required for full stabilization as confirmed by the sixth trace in figure 3(a). Figure 3(b) shows the similar situation but assuming an improved confinement factor $\mathrm{HH}=1.2$. It should be noted that this only changes the $Q$ values. The former example still stabilizes at $19.5 \mathrm{MW}$, but this time at a $Q$ value of 8.8 instead of 7 . The latter effect can be inferred from figure 1 as well.

Most cases shown in figure 3(a) have a slight $Q$ increase with increasing $P_{\mathrm{EC}}$, until near full stabilization where $Q\left(P_{\mathrm{EC}}\right)$ increases rapidly. With $\mathrm{HH}=1.2$, many cases have actually $Q$ decreasing first until near full stabilization. In such cases the best is either full stabilization or even to keep the mode as such with no EC power, in particular for $\mathrm{HH}>1$. These behaviors can be understood from figure $4(a)$ which shows the predicted $w_{\text {sat }}$ versus $P_{\mathrm{EC}}$ for these eight cases. We see that the island width does not change much at first with small values of $P_{\mathrm{EC}}$. Therefore, there is little gain in confinement and a possible loss due to the additional auxiliary power injected. However, when $w_{\text {sat }}$ reduces to near $15 \mathrm{~cm}$, there is a rapid variation with increasing ECCD. This is because the effective $w_{\text {marg }}$ with the $\Delta_{\text {cd }}^{\prime}$ term is around $10 \mathrm{~cm}$ although we have $w_{\text {marg }}=2 \mathrm{~cm}$ in the model.

One case has a different behavior, the dashed line in figure 3, which is the case with $w_{\mathrm{cd}}=5 \mathrm{~cm}, \mathrm{CW}$ and equation (12) (polarization model). In this case, there is a local

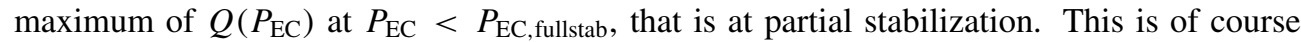
due to a different dependence of $w_{\text {sat }}$ on $P_{\mathrm{EC}}$ as seen from figure 4(a), the dashed line. In this case, $w_{\text {sat }}$ decreases rapidly at an intermediate power. To better understand the origin of this behavior, we plot the right-hand side of equation (12) with increasing $P_{\mathrm{EC}}$ from 0 to $30 \mathrm{MW}$ (figure $4(b)$ ). With increasing $\Delta_{\text {cd }}^{\prime}$, the growth rate has a relatively flat dependence on $w$ for $w>5 \mathrm{~cm}$. This is why for a small power increase, for example between 10 and $14 \mathrm{MW}, w_{\text {sat }}$ shrinks from 15 to $5 \mathrm{~cm}$ (solid circles), yielding a confinement degradation $\Delta_{\tau} w_{\text {sat }} / a$ of $15 \%$ down to $5 \%$ only. On the other hand, since $w_{\text {marg }}$ is small, the peak of the normalized growth rate is still relatively high and one needs an extra $4 \mathrm{MW}$ to shrink $w_{\text {sat }}$ from 5 to $2.5 \mathrm{~cm}$ where it self-stabilizes. These effects could happen more often than expected once all the terms and their various $w$ dependences are taken into account in the modified Rutherford equation. It 

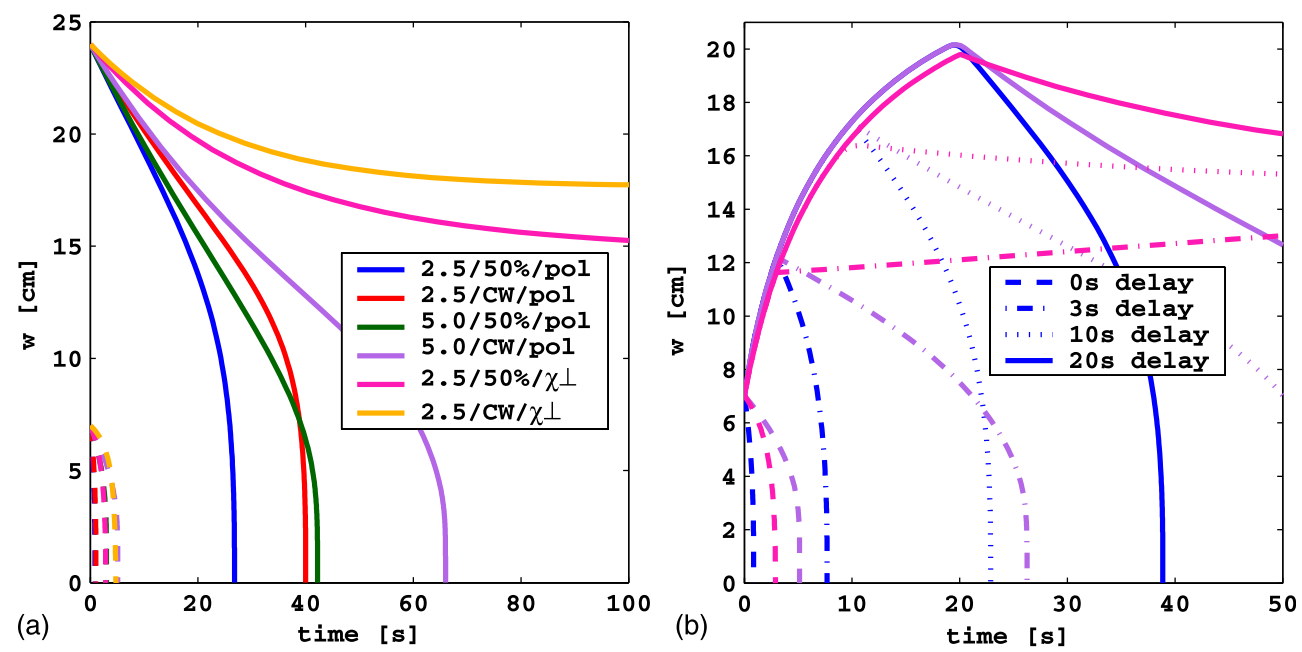

Figure 5. (a) Time evolution predicted for a $2 / 1$ mode in ITER, assuming a $7 \mathrm{~cm}$ and a $24 \mathrm{~cm}$ seed island, using equations (11) and (12) with $13.3 \mathrm{MW}\left(\eta_{\mathrm{NTM}} w_{\mathrm{cd}}=6.3\right)$ and the models indicated in the legend in order of the required $j_{\mathrm{cd}}$ for full stabilization. $(b)$ Using the $1 \mathrm{st}$, 4 th and 5 th models of $(a)$, the time evolution of a $7 \mathrm{~cm}$ seed island with $0 \mathrm{~s}, 3 \mathrm{~s}, 10 \mathrm{~s}$ and $20 \mathrm{~s}$ delay for the CD term to be included.

also shows that the dependence of $w_{\text {sat }}$ on $P_{\mathrm{EC}}$ is neither linear nor smooth, and this could complicate some experimental observations.

\section{Sawteeth and pre-emptive stabilization}

In the previous section, we have seen that it is not always the best option, in terms of global instantaneous performance, to fully stabilize an NTM. In this section we study whether it could be beneficial to turn on the EC power only part of the time, in order to reduce the EC energy injected into the plasma and therefore improve the integrated performance. This depends mainly on the frequency of the trigger mechanism, the size of the seed island and on the time it takes to fully stabilize the mode. It could be that the mode is triggered only in the initial phase of the scenario development, due to a first long sawtooth period or a large first ELM as it is seen in JET. In that case, the best strategy is clearly to fully stabilize the mode, with all the power available and then to turn off the EC power while recovering $Q=10$. Since sawteeth will happen regularly in the stationary ELMy $\mathrm{H}$-mode scenario 2 case, a regular trigger might occur at every sawtooth crash. The sawtooth period can be in between $10 \mathrm{~s}$ and $100 \mathrm{~s}$ in ITER, but is hard to predict, and this will influence the best strategy. On the other hand, the time it takes to fully stabilize the mode can be predicted using equation (11) or (12) with equation (1). This is shown in figure $5(a)$ assuming two seed island sizes of $7 \mathrm{~cm}$ and $24 \mathrm{~cm}$. The first six cases of figure 3 are shown, in order of the required $\left(\eta_{\mathrm{NTM}} w_{\mathrm{cd}}\right)$ values (tables 2 and 3 ), this time for the cases with $w_{\text {marg }}=4 \mathrm{~cm}$. We see that all cases shown can rapidly, within $6 \mathrm{~s}$, fully stabilize the mode with $13.3 \mathrm{MW}$ if the seed island is about $7 \mathrm{~cm}$. However, in two cases (which have $\left(\eta_{\mathrm{NTM}} w_{\mathrm{cd}}\right)>6.3$ in tables 2 and 3 ) the mode cannot be stabilized if the seed island is $24 \mathrm{~cm}$. Note that it still takes between $27 \mathrm{~s}$ and $66 \mathrm{~s}$ to fully stabilize the mode. This can be seen as a reason for the advantage of pre-emptive ECCD (which consists of having EC turned on before the mode is triggered) which will catch the mode in its early stage if it grows from a small island size. Note that the seed island could be created directly at a very large size 
at the sawtooth crash during the reconnection process as observed sometimes in JET [3]. In that case, pre-emptive ECCD would only be beneficial if it modifies the formation of the seed island during the sawtooth crash.

Another aspect to be taken into account to choose the best strategy on ITER is the fact that the resistive time is very long as compared with the present tokamaks. Therefore the NTM growth is very slow, so one might catch the mode early even if the EC power is turned on only after the mode is detected. This is tested in figure $5(b)$ where a seed island of $7 \mathrm{~cm}$ is assumed to be triggered at $t=0$ and then the EC power is turned on at $t=0 \mathrm{~s}, 3 \mathrm{~s}, 10 \mathrm{~s}$ or $20 \mathrm{~s}$. The $0 \mathrm{~s}$ delay would correspond to pre-emptive ECCD, while the other cases to the time it takes to detect the mode, aim at $q=2$ and turn on the power. A $3 \mathrm{~s}$ delay time is a reasonable value if the launchers are already aiming at the $q=2$ surface (from real-time equilibrium and ray-tracing calculations). In this case, three models are displayed, corresponding to the first, fourth and fifth cases shown in figure 5(a) (with corresponding colors). With $0 \mathrm{~s}$ delay, all models can fully stabilize the mode, as seen above. However already with a $3 \mathrm{~s}$ delay, one model cannot stabilize the mode because of the insufficient value of $\left(\eta_{\mathrm{NTM}} w_{\mathrm{cd}}\right)$. If one can fully stabilize the mode, with the other two models shown, then it takes 8-26s if the EC is turned on after $3 \mathrm{~s}, 23-55 \mathrm{~s}$ if there is a delay of $10 \mathrm{~s}$, and $39-75 \mathrm{~s}$ if there is a delay of $20 \mathrm{~s}$. These simulations show that there is a large increase in efficiency if the island is caught when it is below about $10-15 \mathrm{~cm}$. This relates to the effective marginal island size observed in section 5 of about $10 \mathrm{~cm}$ when $\Delta_{\mathrm{cd}}^{\prime}$ is included even if $w_{\mathrm{marg}}=2 \mathrm{~cm}$ in the model. Another result is that it can easily take more than $20 \mathrm{~s}$ to fully stabilize the mode. Therefore, if the sawtooth period is below about $30 \mathrm{~s}$, it is clearly better to keep EC on all the time at either full or partial power depending on the effective $Q$ value obtained. On the other hand, if the sawtooth period is very long, then it might be better to turn on the EC power for a short time of about 10-20 s. Actually, real-time measurements and calculations should allow us to predict the next sawtooth crash and allow the EC to be turned on just before it occurs, yielding the $0 \mathrm{~s}$ delay case of figure $5(b)$. This could become the best strategy if the sawtooth period is longer than $30-60 \mathrm{~s}$. This is even more true if the mode locks when $w<5-10 \mathrm{~cm}$ as predicted in [7]. In that case, it is very important to catch the mode before it grows to that size, although promising stabilization of a locked mode has been recently obtained [26]. From figure 5(b), we see that this means the EC power should be active in less than about $3 \mathrm{~s}$, which means effectively pre-emptive ECCD.

\section{Conclusions}

The plasma performance of a burning plasma, characterized by the factor $Q=P_{\text {fusion }} / P_{\text {aux }}$, is analyzed in detail with respect to the presence of NTMs. A finite island width leads to a locally enhanced radial transport and to a finite confinement degradation. This effect is proportional to $\rho_{\mathrm{s}}^{3}$, where $\rho_{\mathrm{s}}$ is the radius of the $q=m / n$ surface (equations (1)). Therefore the $2 / 1$ mode, with $\rho_{\mathrm{s}} \approx 0.8$, is the most critical and we have concentrated our study on this mode.

The NTMs can effectively be stabilized by driving local current density within the island [9-12]. This shrinks the saturated island and the plasma recovers better confinement. On the other hand, the additional auxiliary power decreases $Q$ (figure 1), except if $P_{\text {fusion }}$ increases significantly. The effective dependence of $Q$ on $P_{\mathrm{EC}}$ for the $2 / 1$ mode on ITER, assuming exact aiming and no mode locking, has been calculated (section 5). It is shown that in most cases there is no much gain, or even a decrease in $Q$, at low $P_{\mathrm{EC}}$, up to near full stabilization. On the other hand, in some cases partial stabilization can lead to better $Q$ factor. This is due to a rapid reduction in the island width with small input power, while the peak of the island growth rate, at a small island width, still needs significant power to be decreased below zero. 
This 'non-linear' dependence of $w_{\text {sat }}$ on $P_{\mathrm{EC}}$ could be observed in the present experiments where more stabilizing terms are at play with similar amplitude and different $w$ dependence. This effect could play a role and complicate comparison of experimental results across machines.

A key element in these discussions is of course the power required for full stabilization. We have analyzed the 2/1 mode predicted in scenario 2 of ITER with respect to two model equations, three different current drive widths, $w_{\mathrm{cd}}$, and three different expected marginal island widths. These model equations, equations (11) and (12), are proposed to be used to compare with the present experimental results. They have been written such that only three main parameters $\left(w_{\text {sat } \infty}, w_{\text {marg }}\right.$ and $\left.c_{j}\right)$ need to be determined and compared with the experimental situation and such that it is most appropriate for analyzing stationary conditions as obtained experimentally. They represent adequately the driving term (with $w_{\text {sat } \infty}$ ), the plasma stabilizing term $\left(w_{\text {marg }}\right)$ and the effect of EC (with $\left.c_{j}\right)$. Note that even though heating effects, CD effects on $\Delta^{\prime}$ and other terms are not explicitly included, they are taken into account implicitly through these three terms. This is in particular useful for cross-machine comparisons. We have seen that the comparison of the $\Delta_{\mathrm{cd}}^{\prime}$ term with experimental results depends on the model equation used, leading to possibly different matching parameters $c_{j}$ in equation (13).

The required values of $j_{\text {cd }}$ for full stabilization of the $2 / 1$ mode in ITER have been calculated. They are obtained from comparing recent experimental results [19] with equations (11) and (12) and inferring the main parameters $w_{\text {sat } \infty}$ and $w_{\text {marg }}$ for each machine (table 1$)$. The minimum values of $\eta_{\mathrm{NTM}}=j_{\mathrm{cd}} / j_{\mathrm{bs}}$ and $\left(\eta_{\mathrm{NTM}} w_{\mathrm{cd}}\right)$ are provided in tables 2 and 3. This allows us to better check whether a given launcher design is able to fully stabilize the $2 / 1$ mode in ITER. For example, the present design yields $\eta_{\mathrm{NTM}} \approx 2.5$ for a typical $w_{\mathrm{cd}}$ of $2.5 \mathrm{~cm}$, giving a value of $\eta_{\mathrm{NTM}} w_{\mathrm{cd}}=6.3$ with $13.3 \mathrm{MW}$. The tables show that this is sufficient to fully stabilize the mode, even with $w_{\mathrm{cd}}=5 \mathrm{~cm}$ to take into account some misalignments of the various beams, especially if $20 \mathrm{MW}$ are used. It also shows that a criterion like $\eta_{\mathrm{NTM}} \geqslant 1.2$ is very simple [21] and both $\eta_{\mathrm{NTM}}$ and $\left(\eta_{\mathrm{NTM}} w_{\mathrm{cd}}\right)$ need to be considered as well as the various models. The results presented in tables 2 and 3 lead to the criterion (equation (20)): $w_{\text {cd }} \leqslant 5 \mathrm{~cm}$ and $\eta_{\mathrm{NTM}} w_{\mathrm{cd}} \geqslant 5 \mathrm{~cm}$.

Figure 1 shows that full stabilization with continuous 13.3 or $20 \mathrm{MW}$ leads to a $Q$ of about 7.7 or 7 , respectively. Therefore, the best strategy cannot be to keep the full power required to stabilize the mode turned on continuously. The analysis of the time it takes to fully stabilize the mode depending on the seed island width and depending on the delay before the ECCD is turned on at the island location helps to determine the best strategies for NTM control. We have shown that if the mode is captured before it reaches about $10 \mathrm{~cm}$, then it is much easier to stabilize the mode and much faster. It can be performed in 3-10s, depending on the model. However with a 3-10 s delay or if the mode has reached about $20 \mathrm{~cm}$, then it can easily take up to $60 \mathrm{~s}$ to get rid of the mode. Note that it takes $3 \mathrm{~s}$ for a mode triggered at a seed island size of $7 \mathrm{~cm}$ to reach $12 \mathrm{~cm}$ (figure 5). This is why pre-emptive ECCD coupled with real-time calculations to determine the time of the next sawtooth crash might be the best strategy to minimize the integrated EC power necessary for NTM control. On the other hand, this will depend on the sawtooth period itself and part of the power might be used to control the sawtooth period. It has been shown in [41] that the sawtooth period can be reduced by about $30 \%$ with ECCD well inside the $q=1$ surface, while it can be increased by $50 \%$ with a real-time control algorithm, similar to NTM control, such that the current is driven just outside the $q=1$ surface with the upper launcher. Thus a combination of sawtooth control and NTM pre-emptive control might be the best. Note that the role of pre-emptive ECCD on the effective size of the seed island triggered at a sawtooth crash after a long sawtooth period has not been studied experimentally yet. This is important to know in order to predict the seed island sizes 
expected on ITER. The goal of stabilizing/controlling the mode before it reaches $10 \mathrm{~cm}$ is also in line with the critical width above which the mode is expected to lock [7]. If it locks, the excursions in confinement (and $\beta, l_{i}$ ) will be probably very large in a burning plasma and a machine like ITER or DEMO and should be avoided. Therefore, pre-emptive ECCD with real-time control to determine when EC will be necessary appear again as the best strategy in order to maximize $Q$. On the other hand, for the hybrid and advanced scenarios, the main source of NTMs is due to the $q$ and pressure profiles evolution. Therefore in these cases, the EC power might need to be turned on continuously to avoid NTMs and the discussion related to figure 1 describes the operational diagram for these scenarios.

The studies presented here show that the requirements to control NTMs in burning plasmas are not just a question of sufficient local current drive capabilities and of aiming at the right position. The role of $P_{\mathrm{EC}}$ on $Q$, the frequency of the trigger events, the size of the seed island, the time it takes to fully stabilize the mode and even the dependence of the driving and stabilizing terms on the island width play a role in determining the best strategy for NTM control.

CEuratom 2010.

\section{References}

[1] Progress in the ITER Physics Basis 2007 Nucl. Fusion 47 S1

[2] Sauter O et al 1997 Phys. Plasmas 41654 and references therein

[3] Sauter O et al 2002 Plasma Phys. Control. Fusion 441999

[4] Chang Z and Callen J D 1990 Nucl. Fusion 2219

[5] Buttery R J et al 2004 Proc. 20th IAEA Conf. on Fusion Energy Conf. (Vilamoura, Portugal, 2004) (Vienna: IAEA) paper IAEA-CN-116/EX/7-1

[6] Hender T C et al 2004 Nucl. Fusion 44778

[7] La Haye R et al 2009 Nucl. Fusion 49045005

[8] Maraschek M et al 2003 Plasma Phys. Control. Fusion 451369

[9] Gantenbein G et al 2000 Phys. Rev. Lett. 851242

[10] La Haye R J et al 2002 Phys. Plasmas 92051

[11] Isayama A et al 2007 Nucl. Fusion 47773

[12] Maraschek M et al 2007 Phys. Rev. Lett. 98025005

[13] Hegna C C and Callen J D 1997 Phys. Plasmas 42940

[14] Zohm H 1997 Phys. Plasmas 44333

[15] Perkins F W et al 1997 Proc. 24th European Conf. on Controlled Fusion and Plasma Physics (Berchtesgaden, Germany, 1997) (Petit Lancy: European Physical Society) vol 21A (ECA) p 1017

[16] Giruzzi G et al 1999 Nucl. Fusion 39107

[17] Ramponi G et al 1999 Phys. Plasmas 63561

[18] Sauter O 2004 Phys. Plasmas 114808

[19] La Haye R et al 2006 Nucl. Fusion 46451

[20] Zohm H et al 2006 14th Workshop on ECE and ECRH (Santorini, Greece)

[21] Saibene G et al 2006 Proc. 21 st IAEA Conf. on Fusion Energy Conf. (Chengdu, China, 2006) (Vienna: IAEA) paper IT/P2-14

[22] Henderson M A et al 2006 Proc. 21st IAEA Conf. on Fusion Energy Conf. (Chengdu, China, 2006) (Vienna: IAEA) paper IT/P2-15

[23] Sauter O et al 2002 Phys. Rev. Lett. 88105001

[24] Porcelli F et al 1996 Plasma Phys. Control. Fusion 382163

[25] Eriksson L-G et al 2004 Phys. Rev. Lett. 92235004

[26] Volpe F A G et al 2009 Phys. Plasmas 16102502

[27] Sauter O et al 2006 Proc. 21st IAEA Conf. on Fusion Energy Conf. (Chengdu, China, 2006) (Vienna: IAEA) paper TH/P3-10

[28] Reimerdes H et al 2002 Phys. Rev. Lett. 88105005

[29] Carrera R et al 1986 Phys. Fluids 29899 
[30] Fitzpatrick R 1995 Phys. Plasmas 2825

[31] Mikhailovskii A B 2003 Contrib. Plasma Phys. 43125 and references herein

[32] Lütjens H, Luciani J-F and Garbet X 2001 Phys. Plasmas 84267

[33] Poli E et al 2002 Phys. Rev. Lett. 88075001

[34] La Haye R J and Sauter O 1998 Nucl. Fusion 38987

[35] Huysmans G T A et al 1999 Nucl. Fusion 391965

[36] Buttery R J et al 2000 Plasma Phys. Control. Fusion 42 B61

[37] Buttery R et al 2002 Phys. Rev. Lett. 88

[38] Westerhof E et al 2007 Nucl. Fusion 4785

[39] La Haye R J et al 2008 Nucl. Fusion 48054004

[40] Ramponi Get al 2008 Nucl. Fusion 48054012

[41] Zucca C 2009 Modeling and control of the current density profile in tokamaks and its relation to electron transport PhD Thesis No. 4360, EPFL, Lausanne http://library.epfl.ch/theses/?nr=4360 